\title{
Gas identification based on bias induced hysteresis of a gas-sensitive SiC field effect transistor
}

\author{
M. Bastuck ${ }^{1}$, C. Bur ${ }^{1,2}$, A. Lloyd Spetz ${ }^{2}$, M. Andersson ${ }^{2}$, and A. Schütze ${ }^{1}$ \\ ${ }^{1}$ Lab for Measurement Technology, Saarland University, Saarbrücken, Germany \\ ${ }^{2}$ Div. of Applied Sensor Science, Linköping University, Linköping, Sweden
}

Correspondence to: M. Bastuck (m.bastuck@1mt.uni-saarland.de)

Received: 26 November 2013 - Accepted: 4 January 2014 - Published: 17 January 2014

\begin{abstract}
In this work dynamic variation of gate bias is used on a gas-sensitive SiC field effect transistor ("GasFET") to optimize its sensitivity and increase its selectivity. Gate bias ramps introduce strong hysteresis in the sensor signal. The shape of this hysteresis is shown to be an appropriate feature both for the discrimination of various gases (ammonia, carbon monoxide, nitrogen monoxide and methane) as well as for different gas concentrations (250 and $500 \mathrm{ppm}$ ). The shape is very sensitive to ambient conditions as well as to the bias sweep rate. Thus, the influences of oxygen concentration, relative humidity, sensor temperature and cycle duration, i.e., sweep rate, are investigated and reasons for the observed signal changes, most importantly the existence of at least two different and competing processes taking place simultaneously, are discussed. Furthermore, it is shown that even for very fast cycles, in the range of seconds, the gas-induced shape change in the signal is strong enough to achieve a reliable separation of gases using gate bias cycled operation and linear discriminant analysis (LDA) making this approach suitable for practical application.
\end{abstract}

\section{Introduction}

Lundström et al. (2007) reported in 1975 for the first time that a field effect transistor with a palladium gate is sensitive to hydrogen. Hydrogen adsorbs and dissociates into ions on the palladium coated gate. The protons diffuse rapidly through the dense metal film and form a polarized layer at the metaloxide interface. This changes the electric field in the oxide, giving rise to a change in the carrier density, which can be measured by the conductivity between drain and source, i.e., in the conducting channel of the transistor.

From 1975 up to now, these sensors have undergone substantial development. In addition to silicon, silicon carbide (SiC) is now preferably used as substrate due to its wide band gap allowing operating temperatures up to $800^{\circ} \mathrm{C}$. This and the chemical inertness of $\mathrm{SiC}$ make SiC-GasFETs (gassensitive $\mathrm{SiC}$ field effect transistor) suitable for operation in harsh environments, e.g., directly in the exhaust stream of combustion engines (Lundström et al., 2007; Lloyd Spetz et al., 2013).
The gate material is usually a catalyst like iridium, platinum or palladium. Regarding the detection, under normal sensor operating conditions, of gases that contain no or strongly bound hydrogen a porous catalyst, as compared to a dense film, is generally required. For any sensor mechanism not involving diffusion/transport of species, e.g., hydrogen, through the gate material, a dense metal film will screen the effect from gas induced charge transfer at the gate material surface, preventing the modulation of the FET channel conductance. A polar molecule, such as carbon monoxide (CO), can for a porous film adsorb directly on or spill over from the metal to the oxide and form a polar layer. The reduction of a surface platinum oxide and an increased sensitivity to background hydrogen has, however, also been proposed as mechanisms taking part in the sensing of $\mathrm{CO}$ by FET devices (Nakagomi et al., 1997; Darmastuti et al., 2011). Ammonia $\left(\mathrm{NH}_{3}\right)$, on the other hand, dissociates at the three phase boundaries (gas, metal, oxide) introduced by the porous gate, whereupon the protons cover the oxide surface or diffuse to the metal-oxide boundary (Lundström et al., 2007; Lloyd Spetz et al., 2013). In addition, 
Schalwig et al. (2002) suggested a spillover of adsorbed oxygen ions as a possible explanation for the detection of nonpolar, (non-)hydrogen containing gases. The negatively charged oxygen ions on the gate oxide influence the electric field, hence the channel conductivity, and can be removed by reducing gases (e.g., $\mathrm{CO}$; nitrogen monoxide, $\mathrm{NO}$; or methane, $\mathrm{CH}_{4}$ ) similar to the sensing mechanism of resistive type metal oxide gas sensors. $\mathrm{CO}, \mathrm{NO}$ and $\mathrm{CH}_{4}$ are chosen as test gases since they are present in the exhaust stream of a combustion engine. The amount of $\mathrm{NO}_{\mathrm{x}}$ can be reduced by adding $\mathrm{NH}_{3}$ (in the form of urea) before the catalytic converter in selective catalytic reduction (SCR) systems. Ideally, there should be no hazardous $\mathrm{NH}_{3}$ left after the catalytic reduction. Exact control of an SCR system thus requires selective detection and quantification of relevant exhaust gas components. Thus, the four mentioned gases were chosen for testing the potential of gate bias cycled operation (GBCO) for sensitive and selective detection in various background gases, e.g., pure nitrogen and air with and without humidity.

Chemical gas sensors in general suffer from poor selectivity. Lee and Reedy (1999) have reviewed the potential of temperature cycled operation (TCO) to increase the selectivity of semiconductor gas sensors. Regarding sensitivity, which changes nonlinearly with the temperature, cyclic operation implicitly includes the point of highest sensitivity for each gas in a given mixture thus optimizing sensitivity. Bur et al. (2012b) extended this approach to GasFETs. In addition to temperature changes, FET-type sensors also allow changing of the gate bias. For solid electrolyte amperometric $\mathrm{NO}_{\mathrm{x}}$ sensors, Schmidt-Zhang et al. (2000) have already shown that different biases can effectively be applied to influence surface reactions and thus the sensor selectivity. Nakagomi et al. (2005) have studied the influence of a constant gate bias on the sensing properties. They found a change in the threshold voltage as well as in sensitivity depending on the gate bias voltage. Thus, cyclic changing of the gate bias again optimizes the sensitivity for all gases and furthermore leads to an enhancement of the selectivity (Bur et al., 2012a), similar to TCO.

Changing the gate bias dynamically, however, leads to strong hysteresis effects (Bastuck et al., 2013), an effect also observed for other transistor type sensors (Petit et al., 2008). Paska and Haick (2012) reported that the hysteresis is strongly affected by trapping and release of charges mostly occurring in the underlying oxide layer of the FET. Usually, this is unwanted and much effort is spent on trying to reduce the hysteresis (Mattmann et al., 2009). However, Schütze et al. (2004) have shown for metal oxide semiconductor (MOS) sensors that the occurrence of hysteresis also means that there is additional information in the transient behavior of the sensor, which can be used to increase selectivity.

In this paper, we study the potential of extracting information from the hysteresis curves obtained by GBCO for improving the sensor performance in terms of selectivity, but also to achieve a better understanding of the various processes occurring on the sensor surface. For demonstrating the potential of this novel method, fast gate bias cycling was also combined with temperature variation as proposed by Bur et al. (2013).

\section{Experimental setup}

\subsection{Sensor device and sensor hardware}

A depletion type SiC GasFET (SenSiC AB, Kista, Sweden) with porous platinum gate (Fig. 1a, b), as described by Andersson et al. (2013), was used for all measurements presented here. The sensor die is mounted on a ceramic heater (Heraeus GmbH, Hanau, Germany) and the temperature is controlled by a Pt100 temperature sensor (Heraeus $\mathrm{GmbH}$, Hanau, Germany) located next to it. For sensor control and data acquisition a combined system developed by $3 \mathrm{~S} \mathrm{GmbH}$, Saarbrücken, Germany, was used. The temperature is controlled by an analog circuit with an accuracy of $1{ }^{\circ} \mathrm{C}$. The gate bias can be set from $-7 \mathrm{~V}$ to $+7 \mathrm{~V}$ using an 8 bit DAC, i.e., with a resolution of approximately $50 \mathrm{mV}$. Data acquisition is performed with a 10 bit ADC for measuring the drainsource current with a measurement range of $0-1000 \mu \mathrm{A}$, resulting in a resolution of approximately $1 \mu \mathrm{A}$. The acquisition rate for all measurements was $10 \mathrm{~Hz}$. The recorded sensor signal is the drain-source current $I_{\mathrm{DS}}$ at constant voltage $V_{\mathrm{DS}}=2 \mathrm{~V}$. Figure 2 shows typical IV curves of the tested devices showing the dependence of the sensitivity (here highest at $V_{\mathrm{GS}}=2 \mathrm{~V}$ ) and selectivity (here highest at $V_{\mathrm{GS}}=0 \mathrm{~V}$ ) on the gate bias at $200{ }^{\circ} \mathrm{C}$ in dry air. The constant drain-source voltage of $V_{\mathrm{DS}}=2 \mathrm{~V}$ was chosen because this marks the onset of the saturation region at $V_{\mathrm{GS}}=0 \mathrm{~V}$ without gas and thus provides a large dynamic range, which is important for dynamic operation of gas sensors.

\subsection{Measurement setup}

In order to study the influence of the gate bias the sensor was exposed to several, typical exhaust gases under various conditions. As test gases $\mathrm{CO}, \mathrm{NO}$ and $\mathrm{CH}_{4}$ as well as $\mathrm{NH}_{3}$ were chosen; each test gas was tested at a concentration of $500 \mathrm{ppm}$, and for $\mathrm{NH}_{3}$ and $\mathrm{CO}$ additionally at $250 \mathrm{ppm}$. Measurements were carried out in pure nitrogen and in synthetic air, both in dry and humid (50\% relative humidity, $\mathrm{RH})$ atmospheres in order to study the influence of oxygen and water vapor. The sensor was mounted in a stainless steel test chamber with a volume of $3 \mathrm{~mL}$ and the total flow over the sensor was kept constant at $100 \mathrm{~mL} \mathrm{~min}^{-1}$.

The sensor was heated to operating temperatures of 118 , 187 and $265^{\circ} \mathrm{C}$ respectively, to study the influence of the operating temperature. The elevated temperature is necessary to provide sufficient energy for the dissociation and/or reaction of the gas molecules and, thus, making the FET gas sensitive. Furthermore, Bur et al. (2012b) pointed out that temperature 


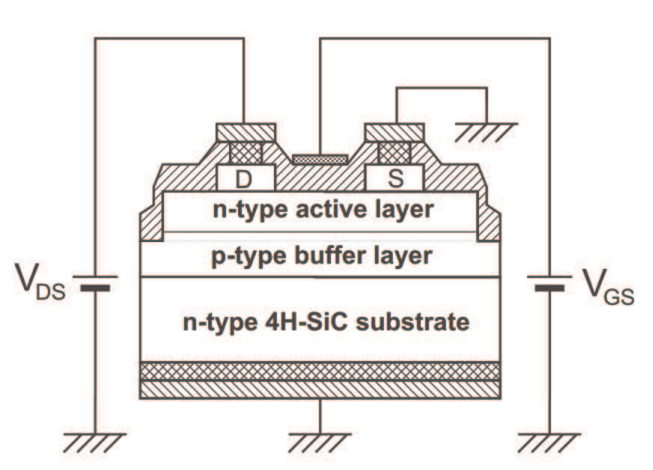

(a)

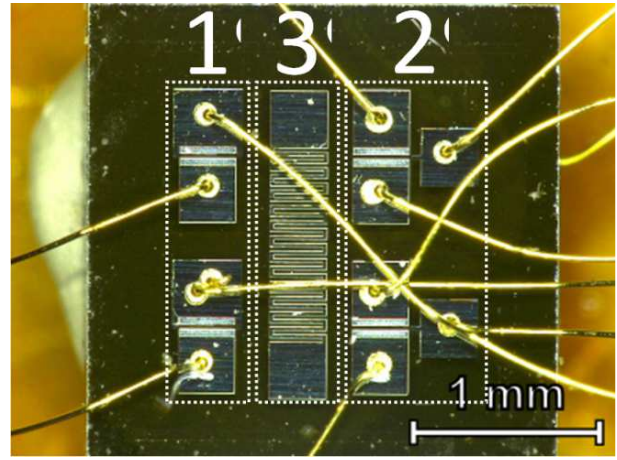

(b)

Figure 1. Schematic cross-sectional view of the studied MISiC-FET (a), and top view of the sensor chip comprising four sensor devices (1: gate connected to drain, 2: variable gate) and a Pt100 temperature sensor (3) (b).

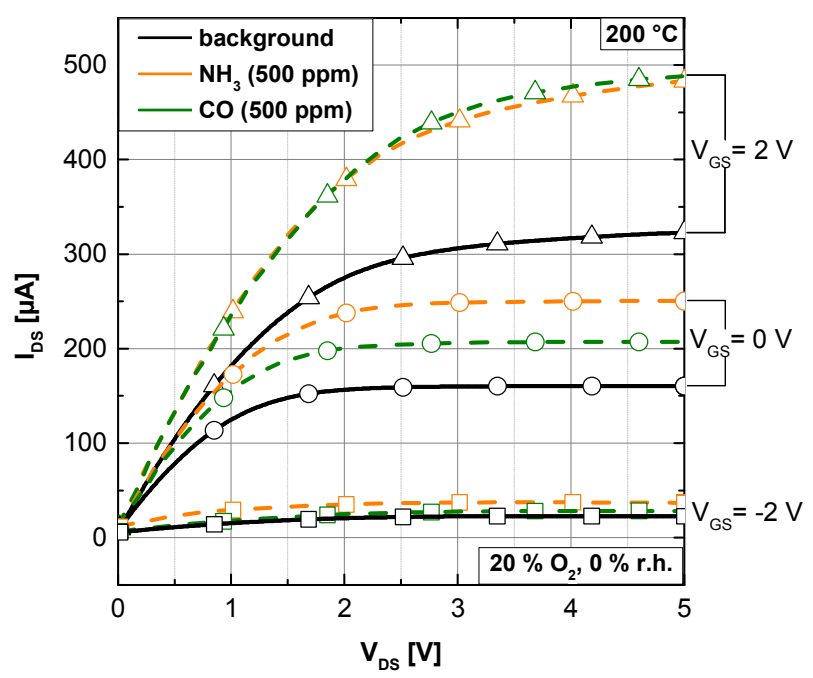

Figure 2. Typical IV curves of the sensor in air (black) and test gases. The sensitivity towards ammonia (orange, dashed) and CO (green, dashed) changes nonlinearly with the applied gate potential, which can either be used to maximize the sensor response for one distinct gas or to identify a gas by a gate bias sweep.

strongly influences the sensitivity for the target gases, which was considered in the choice of temperatures.

In order to study hysteresis curves the gate bias was linearly ramped up from $-3 \mathrm{~V}$ to $+7 \mathrm{~V}$ and back down again over $900 \mathrm{~s}$ each (Fig. 3a). Before and after these ramps, the potential was kept constant at $-3 \mathrm{~V}$ for $300 \mathrm{~s}$ (in background) or $30 \mathrm{~s}$ (in test gas) each. This cycle was repeated three times at each temperature in background as shown in Fig. 3b. One cycle was run at each temperature in every test gas (Fig. 3c).Temperature changes were only made between two cycles, thus, the device was given sufficient time to reach equilibrium before a new gate bias ramp was started. Before each measurement series, i.e., gas composition change, the sensor was run in background only for at least one hour, and subsequently in the investigated gas composition until the baseline had stabilized.

Additionally, the influence of the bias sweep rate was studied by testing ramps with a duration of $300 \mathrm{~s}$ and $60 \mathrm{~s}$, respectively. Varying sweep rates offer the possibility to estimate time constants of phenomena involved in the sensor response mechanisms, possibly providing information helpful in the separation of different, competing effects.

\subsection{Data evaluation}

The recorded raw signals plotted vs. the applied gate bias $V_{\mathrm{GS}}$ are shown in Fig. 4a and $\mathrm{c}$ for two different temperatures. In order to suppress the sensor signal variation in the background gas, which actually shows nearly no hysteresis, and to enhance the gas induced signal changes the difference signal $\Delta I_{\mathrm{DS}}=I_{\mathrm{DS}}$ (gas) $-I_{\mathrm{DS}}$ (background) is used for further analysis (Fig. 4b, d). The background signal was determined from the mean value of three gate bias cycle measurements. Although the first cycle usually shows slightly lower values, the background cycles exhibit negligible variations thus showing good overall reproducibility (Fig. 3d). The same applies to background cycles recorded after and, respectively, between test gas exposures.

To discriminate different gases, a relatively fast cycle is run several times in each of the examined atmospheres, and predefined features are calculated for every cycle. The obtained multi-dimensional data of the so-called virtual multi-sensor (Schütze et al., 2004) is usually evaluated by pattern recognition tools. Instead of the often used principal component analysis (PCA, un-supervised learning), here linear discriminant analysis (LDA) was employed as a powerful tool for evaluation of multivariate data. LDA is a supervised learning method, which means that the correct classification is known for each object, i.e., cycle (Gutierrez-Osuna, 2002; Klecka, 1980). With this method, it is possible to determine whether 


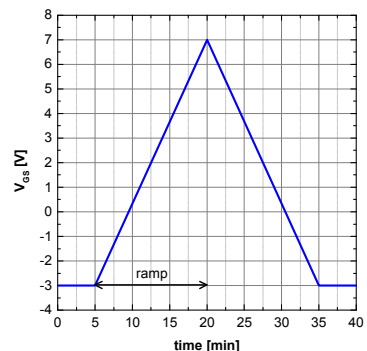

(a)

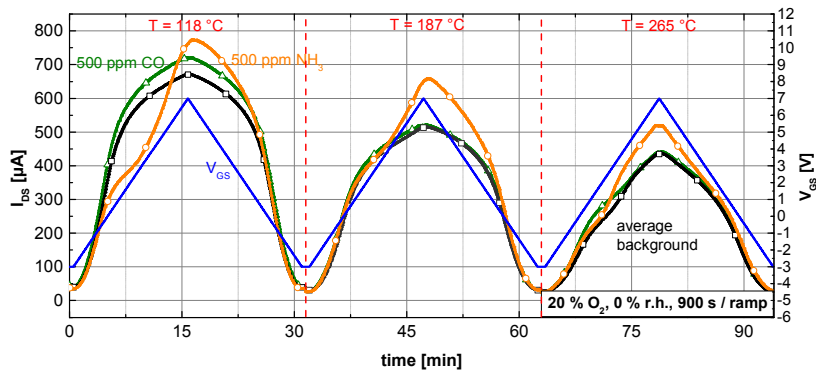

(c)

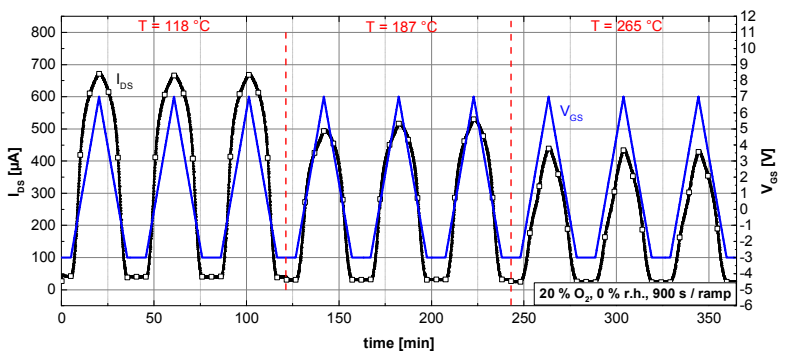

(b)

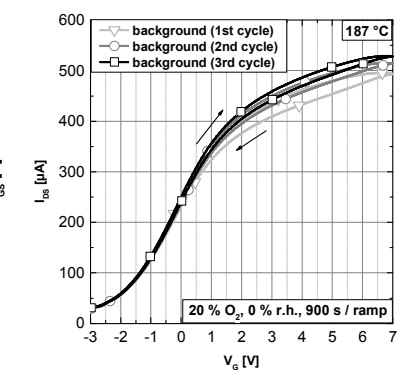

(d)

Figure 3. Visualization of a gate bias cycle (a), an excerpt from an actual measurement showing the gate potential (blue) and sensor response (black) at three different temperatures in background (black, rectangles) (b) and with ammonia (orange, circle) or CO (green, triangles) (c), and the absolute signal of three cycles in background (d). One hysteresis curve consists of one ramp up and one ramp down.

the gas-induced changes in the hysteresis shape are sufficient, i.e., distinct enough and also reproducible, to achieve a nonambiguous classification of the different gases.

In order to describe the shape of a hysteresis generally some easily computable features were defined: the largest horizontal and vertical widths of the hysteresis, the position of these maxima, as well as the area enclosed by the curve (cf. Fig. 12b).

\section{Results and discussions}

\subsection{Gas discrimination}

The measured data show that the shape of the hysteresis signal of a GasFET is a suitable feature for gas discrimination. The gases can be clearly distinguished from background as well as from each other based on the shape of the hysteresis curves (Fig. 4a, c). After subtraction of the background signal, a peak becomes evident for $\mathrm{CO}$ and $\mathrm{NH}_{3}$ (Fig. 4b), which appears around $0 \mathrm{~V}$. Thus, it can be interpreted as a polarity dependent effect, e.g., the flipping of trap states, as postulated by Paska and Haick (2012), or the movement of mobile oxide charges. The transfer characteristics inherent to the device (as discussed in the last paragraph in Sect. 3.4) could contribute to the observed peak as well. At $265^{\circ} \mathrm{C}$, this peak is even more distinct and the hysteresis width is smaller, especially for $\mathrm{CO}$, than at $187^{\circ} \mathrm{C}$ (Fig. 4d). More- over, a crossover point appears for both gases around $2.5 \mathrm{~V}$, which indicates that there are two different and competing processes, e.g., surface reactions and/or diffusion, influencing the sensor signal.

Figure 5 a shows the hysteresis with background subtracted for $\mathrm{NO}$ and $\mathrm{CH}_{4}$ at $187^{\circ} \mathrm{C}$ sensor temperature. Comparison with Fig. $4 \mathrm{~b}$ reveals obvious differences in the signal shapes of all four gases, thus allowing identification of all four gases. No peaks appear around the $0 \mathrm{~V}$ gate bias for $\mathrm{NO}$ and $\mathrm{CH}_{4}$; instead, a change in slope is observed for $\mathrm{NO}$, while $\mathrm{CH}_{4}$ shows a crossover point of the signals for ramp up and ramp down. Regarding the sensor temperature (cf. Fig. 5a and b), a similar change as for $\mathrm{CO}$ and $\mathrm{NH}_{3}$ can be observed: at $265^{\circ} \mathrm{C}$, the difference signal $\Delta I_{\mathrm{DS}}$ and, thus, the sensitivity are reduced. A significant sensor response is only observed for a gate bias above $3 \mathrm{~V}$. Crossover points are now observed for both gases, although at different gate bias voltages, while the hysteresis width again decreases.

\subsection{Influence of oxygen and humidity}

Figure 6 shows the sensor signals $\Delta I_{\mathrm{DS}}$ for $\mathrm{CO}$ and $\mathrm{NH}_{3}$ in dry air, i.e., at $20 \%$ oxygen $\left(\mathrm{O}_{2}\right)$, in Fig. 7 at $50 \%$ $\mathrm{RH}$ in pure nitrogen, and in Fig. 8 in humid air, i.e., with $20 \% \mathrm{O}_{2}$ plus $50 \% \mathrm{RH}$. All three figures also show significant signal changes resulting from an increase of the 

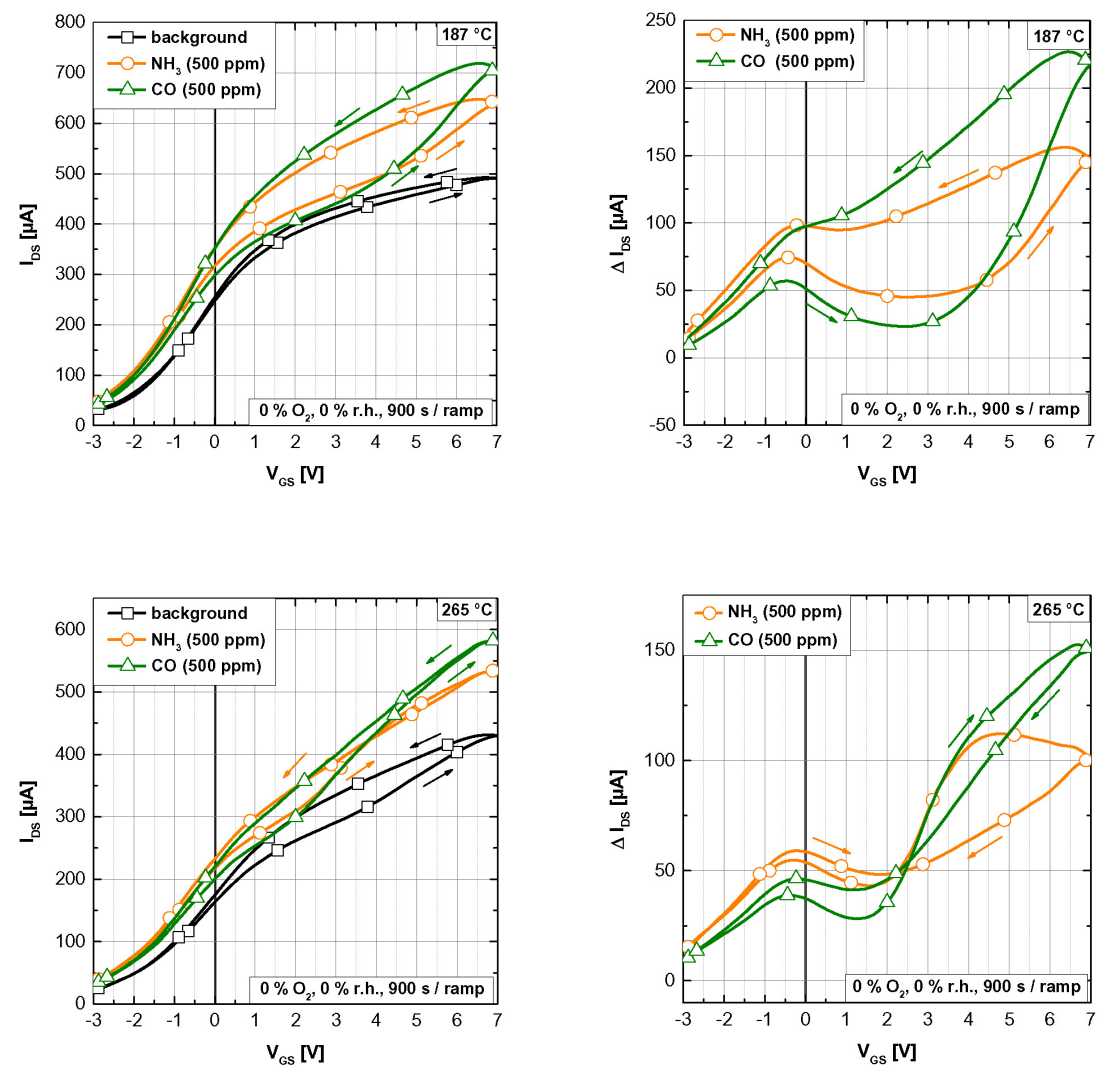

Figure 4. The absolute sensor response (a, c) and the difference signal vs. background $\Delta I_{\mathrm{DS}}=I_{\mathrm{DS}}$ (gas) $-I_{\mathrm{DS}}$ (background) (b, d) for a background of dry nitrogen (black, rectangles) as well as $500 \mathrm{ppm}$ ammonia (orange, circles) and $500 \mathrm{ppm} \mathrm{CO}$ (green, triangles) at $187^{\circ} \mathrm{C}$ (a, b) and $265^{\circ} \mathrm{C}(\mathbf{c}, \mathbf{d})$.

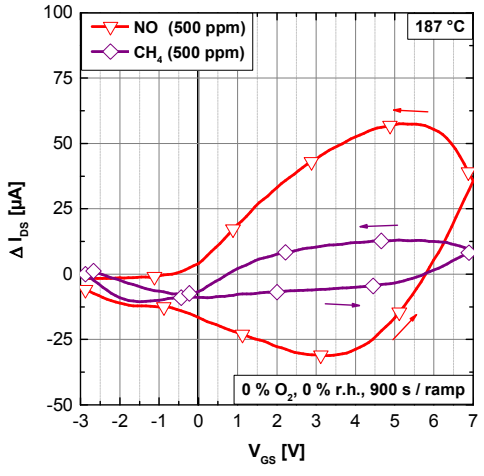

(a)

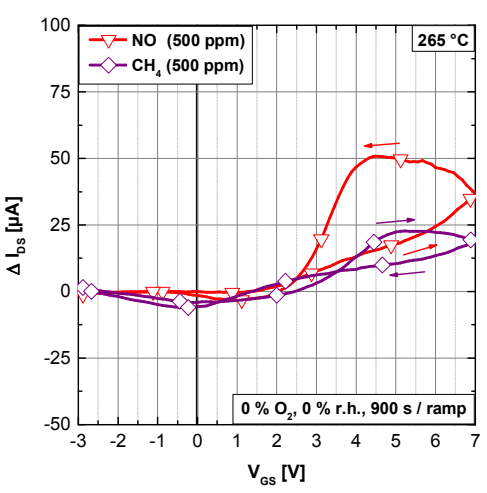

(b)

Figure 5. Hysteresis of $\Delta I_{\mathrm{DS}}$ for $500 \mathrm{ppm} \mathrm{NO}$ (red, triangles) and $\mathrm{CH}_{4}$ (purple, diamonds) in dry nitrogen at $187^{\circ} \mathrm{C}$ (a) and $265^{\circ} \mathrm{C}(\mathbf{b})$. 


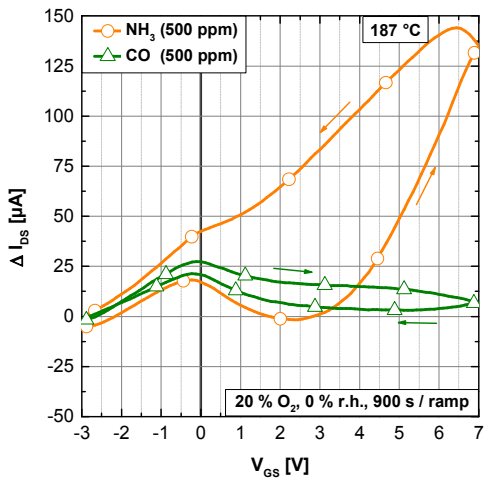

(a)

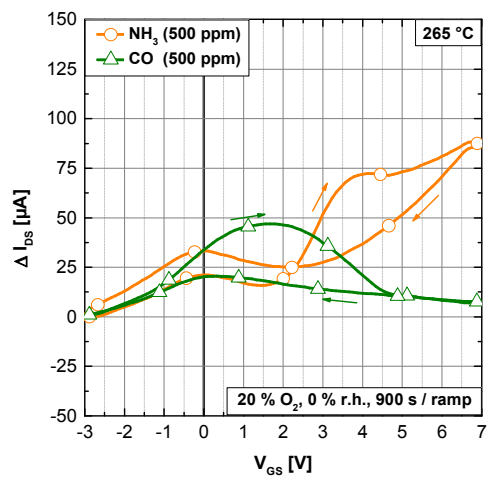

(b)

Figure 6. Hysteresis of $\Delta I_{\mathrm{DS}}$ for $\mathrm{CO}$ (green, triangles) and $\mathrm{NH}_{3}$ (orange, circles) in dry air $\left(20 \% \mathrm{O}_{2}, 0 \% \mathrm{RH}\right)$ at $187^{\circ} \mathrm{C}(\mathbf{a})$ and $265^{\circ} \mathrm{C}(\mathbf{b})$.

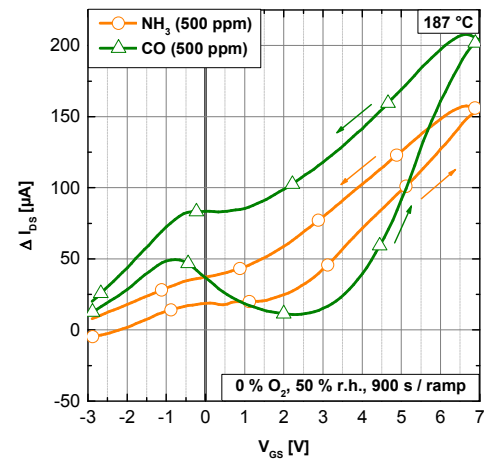

(a)

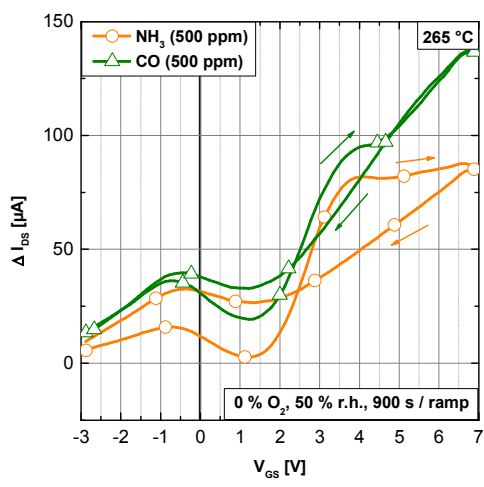

(b)

Figure 7. Hysteresis of $\Delta I_{\mathrm{DS}}$ for $\mathrm{CO}$ (green, triangles) and $\mathrm{NH}_{3}$ (orange, circles) in pure nitrogen at $50 \% \mathrm{RH}$ at $187^{\circ} \mathrm{C}(\mathbf{a})$ and $265^{\circ} \mathrm{C}(\mathbf{b})$.

operating temperature from $187^{\circ} \mathrm{C}$ (Figs. 6a, 7a, 8a) to $265^{\circ} \mathrm{C}$ (Figs. 6b, 7b, 8b).

Background oxygen strongly influences the $\mathrm{CO}$ signal while it has little effect on the $\mathrm{NH}_{3}$ response (cf. Figs. $4 \mathrm{~b}$ and $6 \mathrm{a}$ as well as $8 \mathrm{a}$ ). This could be expected since $\mathrm{CO}$ as a very reactive reducing gas interacts with negatively charged oxygen ions on the sensor surface. At either low temperatures or a high $\mathrm{CO} / \mathrm{O}_{2}$ ratio, the Pt surface is completely reduced, i.e., no adsorbed oxygen is present, whereas it is almost completely covered with adsorbed oxygen for high temperatures or a low $\mathrm{CO} / \mathrm{O}_{2}$ ratio (Johansson et al., 2001). Thus, in the presence of $20 \% \mathrm{O}_{2}$ the equilibrium coverage of $\mathrm{CO}$ on the sensor surface is small, hence both the sensor signal as well as the hysteresis width decrease strongly (Fig. 6a). However, $\mathrm{CO}$ can still be detected due to the peak around the $0 \mathrm{~V}$ gate bias.

In contrast, the hysteresis width decreases strongly for $\mathrm{NH}_{3}$ in the presence of humidity while the $\mathrm{CO}$ signal remains largely unchanged (cf. Figs. $4 \mathrm{~b}$ and 7a). In addition, the $0 \mathrm{~V}$ peak for $\mathrm{NH}_{3}$ vanishes almost completely.
With both oxygen and humidity present in the background, the change in the signal shape upon $\mathrm{CO}$ exposure seems to be dominated by the influence of oxygen at $187^{\circ} \mathrm{C}$ and $265^{\circ} \mathrm{C}$ (cf. Figs. 4 and 7 as well as 6 and 8, respectively). The effect of background on the $\mathrm{NH}_{3}$ response is more complicated: at $187^{\circ} \mathrm{C}$, the hysteresis width in humid air is smaller than in dry nitrogen, which is similar to the response in humid nitrogen; in addition, the peak around $0 \mathrm{~V}$, which is not observed in humid nitrogen, is very pronounced (Fig. 8a). At $265^{\circ} \mathrm{C}$, in addition to a slightly smaller hysteresis width compared to dry oxygen, no clear crossover point is seen, which only happens in this background (Fig. 8b).

The sensor temperature is another parameter that can be used to alter the shape of the hysteresis drastically. Figures $6 \mathrm{~b}$ and $7 \mathrm{~b}$ show the same measurements as in Figs. 6a and 7a, respectively, but with a sensor temperature of $265^{\circ} \mathrm{C}$ instead of $187^{\circ} \mathrm{C}$. As an overall effect for the gases and temperature range investigated here, higher temperature leads to lower signals and, thus, lower sensitivity. Nakagomi et al. (2005) reported that this effect can be compensated by a positive 


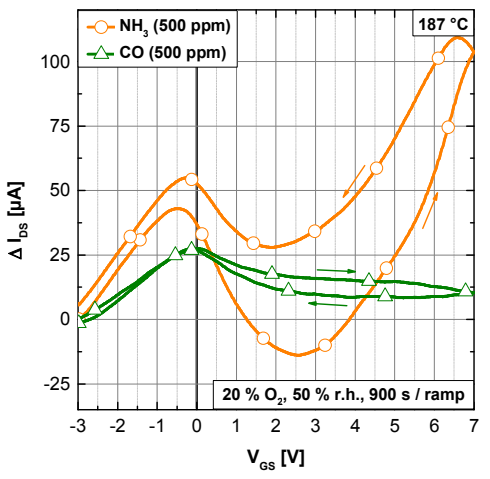

(a)

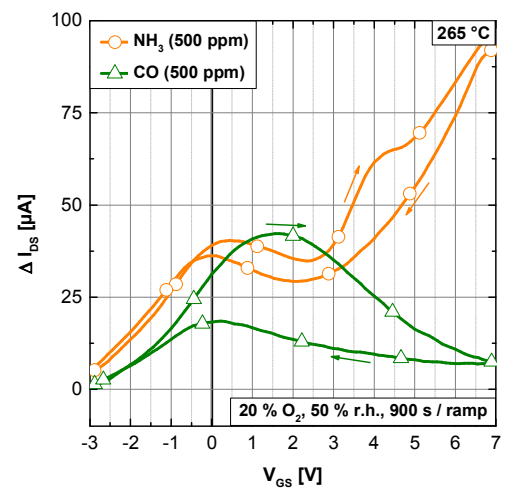

(b)

Figure 8. Hysteresis of $\Delta I_{\mathrm{DS}}$ for $\mathrm{CO}$ (green, triangles) and $\mathrm{NH}_{3}$ (orange, circles) in humid air $\left(20 \% \mathrm{O}_{2}, 50 \% \mathrm{RH}\right)$ at $187^{\circ} \mathrm{C}(\mathbf{a})$ and $265^{\circ} \mathrm{C}$ (b).
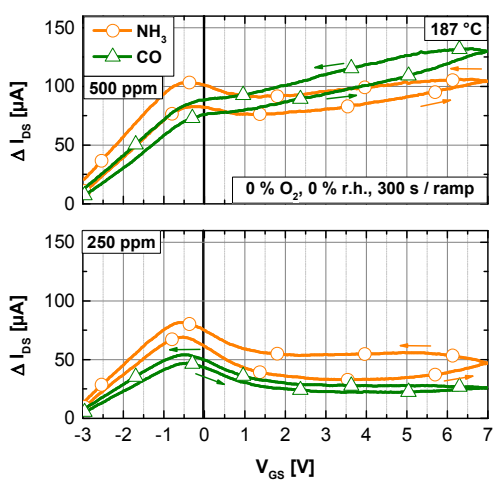

(a)
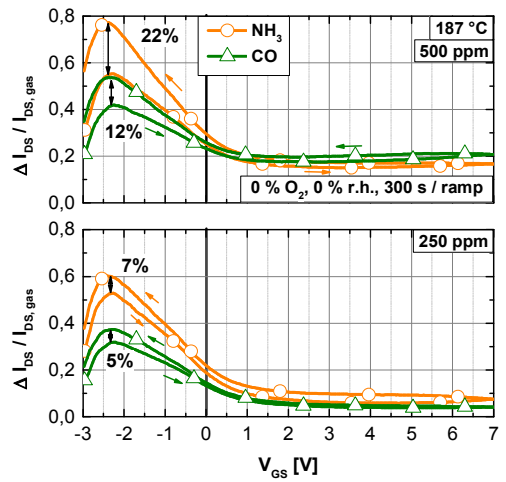

(b)

Figure 9. Influence of the gas concentration on the hysteresis in difference signal (a) and in difference signal relative to the absolute signal in gas (b).

gate bias. In addition, there is a strong change of the signal shape, resulting in greater differences between the gases, i.e., improved selectivity. At this higher temperature, a stronger hysteresis is detected for $\mathrm{CO}$ in air at moderate gate bias values (0-4 V, Figs. 6b, 8b). More significantly, crossover points appear in the hysteresis for $\mathrm{NH}_{3}$ in dry air (Fig. 6b) and for both gases in nitrogen (Figs. 4d, 7b). The position of these crossover points can be used as an additional feature for gas discrimination. They also indicate that two competing processes with different time constants and temperature dependence are occurring on the sensor surface or, more likely, due to the long time constants, which indicate diffusion processes in the sensor layers, e.g., moving charges in the gate oxide.

\subsection{Influence of gas concentration}

In addition to gas identification, it is also possible to determine the gas concentration by analyzing the hysteresis, here exemplarily shown for $\mathrm{CO}$ and $\mathrm{NH}_{3}$. As shown in Fig. 9a, a reduced gas concentration of $250 \mathrm{ppm}$ leads to significant changes especially for the CO response. However, the hysteresis widths show a good correlation to the concentration for both gases. This effect is most evident at low gate bias values and can thus be enhanced by dividing the difference signal $\Delta I_{\mathrm{DS}}$ by the absolute signal in gas, i.e., $\Delta I_{\mathrm{DS}} / I_{\mathrm{DS}}$ (gas), to obtain a relative signal change as shown in Fig. 9 b. Reducing the gas concentration from $500 \mathrm{ppm}$ to $250 \mathrm{ppm}$ results in a decrease of the hysteresis width from $22 \%$ to $7 \%$ for $\mathrm{NH}_{3}$ and from $12 \%$ to $5 \%$ for $\mathrm{CO}$. Note that for these measurements, faster ramps with duration of only $300 \mathrm{~s}$ were used, which also changes the hysteresis significantly (cf. Figs. 4b and $9 \mathrm{a}$ as well as Sect. 3.4 below). However, discrimination of $\mathrm{NH}_{3}$ and $\mathrm{CO}$ (and also $\mathrm{NO}$ and $\mathrm{CH}_{4}$ ) is still possible. 


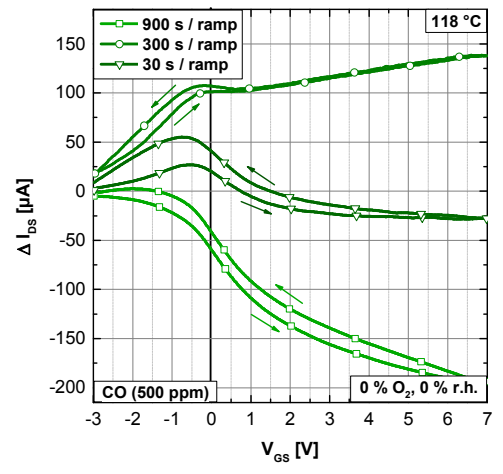

(a)

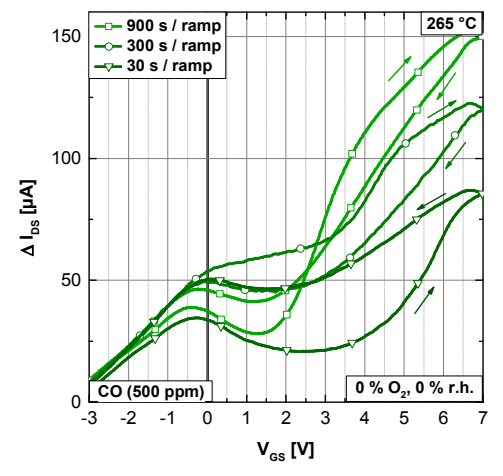

(b)

Figure 10. Hysteresis for $\mathrm{CO}(500 \mathrm{ppm})$ in dry nitrogen at $118^{\circ} \mathrm{C}(\mathbf{a})$ and $265^{\circ} \mathrm{C}$ (b) for different ramp durations (rectangles: $900 \mathrm{~s}$; circles: 300 s; triangles: 30 s).

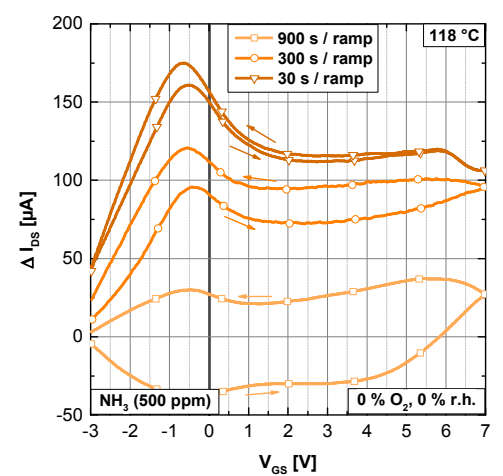

(a)

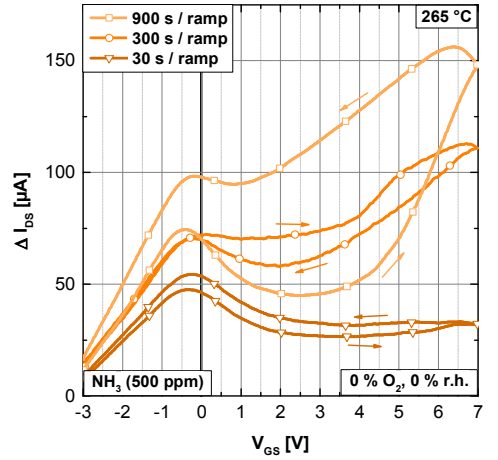

(b)

Figure 11. Hysteresis for $\mathrm{NH}_{3}(500 \mathrm{ppm})$ in dry nitrogen at $118^{\circ} \mathrm{C}$ (a) and $265^{\circ} \mathrm{C}$ (b) for different ramp durations (rectangle marker $900 \mathrm{~s}$, circle marker $300 \mathrm{~s}$, triangle marker $30 \mathrm{~s}$ ).

\subsection{Influence of the gate bias sweep rate}

Obviously, a strong influence of the cycle duration, i.e., the gate bias ramp rate, on the hysteresis could be expected as this is evidence that the sensor is not in equilibrium. Thus, in addition to measurements lasting $30 \mathrm{~min}$ (1800 s corresponding to a ramp rate of $10 \mathrm{~V} / 900 \mathrm{~s}=11.1 \mathrm{mV} \mathrm{s}^{-1}$ ) per cycle, shorter cycle durations, i.e., faster bias ramp rates, of $600 \mathrm{~s}$ $\left(33.3 \mathrm{mV} \mathrm{s}^{-1}\right)$ and $60 \mathrm{~s}\left(333 \mathrm{mV} \mathrm{s}^{-1}\right)$ were evaluated to study the potential of this method for realistic application scenarios requiring shorter response times as shown in Figs. 10 and 11 for $\mathrm{CO}$ and $\mathrm{NH}_{3}$, respectively.

A significant change in the hysteresis shape is observed for shorter cycles, which is another indication of the presence of at least two processes with different time constants. A good example for this effect is the observed behavior for $\mathrm{CO}$ at $118^{\circ} \mathrm{C}$ (Fig. 10a), where the signal becomes negative for the $900 \mathrm{~s}$ ramp, but positive during the $300 \mathrm{~s}$ ramp. For the $30 \mathrm{~s}$ ramp, the signal change is relatively small compared to the two other cases, i.e., almost zero except for a small peak around $0 \mathrm{~V}$, indicating that the processes on the sensor surface at this temperature are too slow to lead to a significant signal change. However, the hysteresis width is highest for the fast ramp, indicating a strong nonequilibrium condition. At the higher sensor temperature of $265^{\circ} \mathrm{C}$ (Fig. 10b), the signal continuously decreases for increasing sweep rates; however, the hysteresis is again more pronounced for the higher ramp rates. Furthermore, the signal is positive for every sweep rate tested; however, a crossover point appears only for the slow $900 \mathrm{~s}$ ramp. This indicates an underlying process, either surface reactions or - more likely - diffusion, with a time constant between $300 \mathrm{~s}$ and $900 \mathrm{~s}$, which is only observed during the long cycle.

For $\mathrm{NH}_{3}$ at $118^{\circ} \mathrm{C}$ (Fig. 11a) the hysteresis width is small at high sweep rates, contrary to the behavior observed for $\mathrm{CO}$. The $30 \mathrm{~s}$ ramp, which produced nearly no signal for $\mathrm{CO}$, shows the highest signal of all tested ramps for $\mathrm{NH}_{3}$, indicating that the process responsible for the $\mathrm{NH}_{3}$ response is fast 


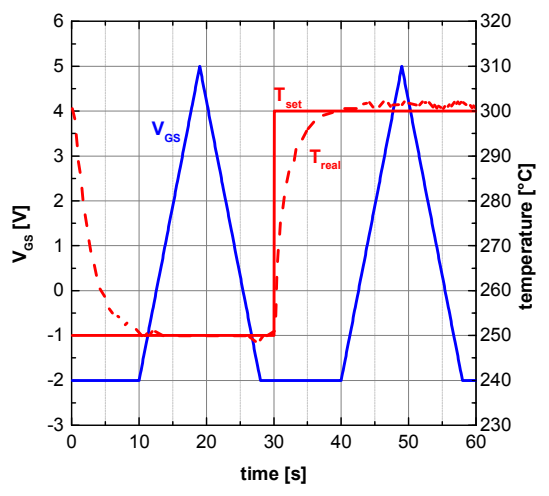

(a)

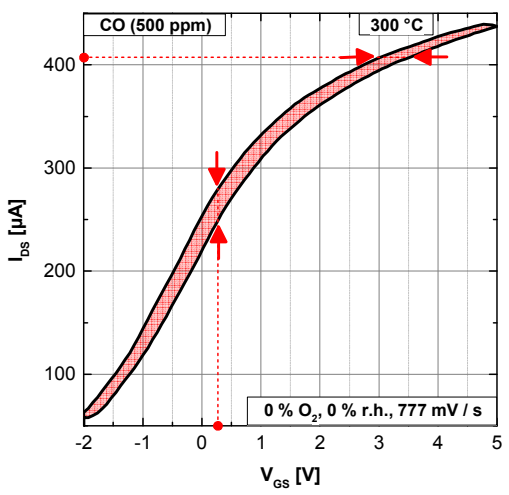

(b)

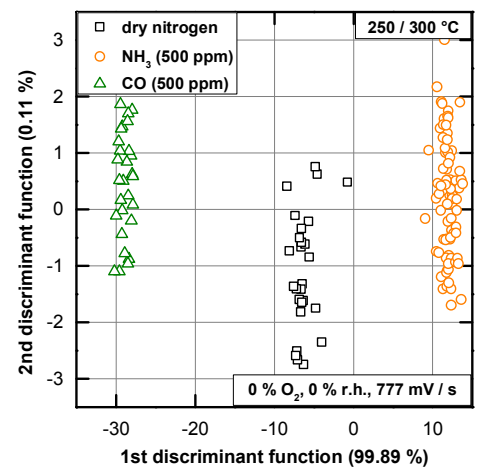

(c)

Figure 12. Combined TCO-GBCO cycle (a). Features that can be extracted from a hysteresis curve: the maximum horizontal and vertical width (red arrows), the position of these maxima (red dots) and the area enclosed by the curve (light red) (b). LDA discrimination between dry nitrogen (background), $\mathrm{CO}$ and ammonia based on hysteresis features (horizontal and vertical width and area) from $9 \mathrm{~s}$ ramps (from $-2 \mathrm{~V}$ to $5 \mathrm{~V}$, i.e., $777 \mathrm{mV} \mathrm{s}^{-1}$ ) at $250^{\circ} \mathrm{C}$ and $300^{\circ} \mathrm{C}$ plus the signal mean value over the interval of $0-10 \mathrm{~s}$ of the cycle (c).

even at this low temperature in an atmosphere of $0 \% \mathrm{O}_{2}$ and $0 \% \mathrm{RH}$. The main cause of the sensor signal observed here is probably $\mathrm{NH}_{3}$ adsorption on $\mathrm{Pt}$ (Wallin et al., 2004), which is favored over $\mathrm{O}_{2}$ adsorption, and consecutive spillover of $\mathrm{NH}_{3}$ to the oxide, thus replacing oxygen anions with polar $\mathrm{NH}_{3}$ molecules. This is also supported by Fogelberg et al. (1987), who reported that $\mathrm{O}_{2}$ is necessary for $\mathrm{NH}_{3}$ to dissociate (results from ultra high vacuum studies at $50-250{ }^{\circ} \mathrm{C}$ and with $\mathrm{Pd}$ as catalytic metal). At $265^{\circ} \mathrm{C}$ (Fig. 11b), both the hysteresis width as well as the signal itself decrease with increasing ramp rate indicating that competing processes, e.g., $\mathrm{NH}_{3}$ desorption, are more relevant at this temperature.

For $\mathrm{NH}_{3}$ (Fig. 11), and, with some restrictions, $\mathrm{CO}$ (Fig. 10) as well, an inversion of the maximum signals for different sweep rates is reached by an increase of the temperature. At $118^{\circ} \mathrm{C}$, the $900 \mathrm{~s}$ ramp produces the lowest signals for $\mathrm{NH}_{3}$ while the $30 \mathrm{~s}$ ramp produces the highest; this relation is inverted at $265^{\circ} \mathrm{C}$. The same applies to $\mathrm{CO}$, with the exception of the $30 \mathrm{~s}$ ramp, which shows almost no signal at $118^{\circ} \mathrm{C}$. Presumably, this behavior is again caused by at least two competing effects with different time constants.

Another effect becomes obvious when comparing Figs. 10a and 11a and Figs. 10b and 11b, i.e., low and high temperatures, respectively: different signal slopes for positive gate bias voltages. While the signal has a very low slope, or is even decreasing (Fig. 10a, 900 s), for all examined ramps at $118^{\circ} \mathrm{C}$, the slope in this region is dramatically higher at $265^{\circ} \mathrm{C}$, especially for low sweep rates. Assuming the same surface/interface conditions upon exposure to a certain gas mixture and the same sensing/transduction mechanisms at play for all gate biases, a negative slope would be expected from simple transistor device characteristics when entering the linear region with increasing gate bias. Thus, the observed positive slope most likely indicates either a gate bias dependent shift of the equilibrium conditions on the sensor surface, e.g., the coverage of oxygen ions (introduced by leakage or impurities) and other surface species such as adsorbed $\mathrm{CO}$ and $\mathrm{NH}_{\mathrm{x}}$, or a change in the sensing/transduction mechanisms with a change in gate bias 
(or both). An increased gate bias could for example lead to stronger binding of electrons in the metal, i.e., a higher energy barrier for the formation of oxygen ions, and/or a change in the equilibrium spillover of oxygen anions from the metal to the silicon dioxide surface.

\subsection{Combination of temperature and gate bias cycle}

Shorter cycles (up to one minute) are required to use the presented method in practical applications similar to temperature cycled operation (TCO). As the hysteresis becomes more distinct for higher sensor temperatures, this can be exploited to achieve shorter cycle times. A simple $T$ cycle consisting of two temperature plateaus $\left(250^{\circ} \mathrm{C}\right.$ and $\left.300^{\circ} \mathrm{C}\right)$, each $30 \mathrm{~s}$ long, was chosen to obtain higher sensitivity (Bur et al., 2012b) and faster response. After $10 \mathrm{~s}$ on each plateau to reach a steady temperature the gate bias was cycled from $-2 \mathrm{~V}$ to $5 \mathrm{~V}$ and back within $9 \mathrm{~s}$ per ramp, corresponding to a ramp rate of $777 \mathrm{mV} \mathrm{s}^{-1}$, as shown in Fig. 12a. This oneminute cycle is suitable to achieve a very clear discrimination of background (dry nitrogen), $500 \mathrm{ppm} \mathrm{CO}$ and $500 \mathrm{ppm}$ $\mathrm{NH}_{3}$, using LDA. The LDA plot shown in Fig. 12c was calculated using only the vertical and horizontal (absolute) maximum width, the areas enclosed by the two hysteresis curves and the signal mean value between $0 \mathrm{~s}$ and $10 \mathrm{~s}$ of the cycle, i.e., a total of seven features, three each for both temperature plateaus and the mean value. The plot shows a total of 136 cycles, thus the number of features is much lower than the number of samples to avoid overfitting. Note that the discrimination would probably also be possible for lower concentrations as the two gases show a shift in opposite directions from the background.

To extend this approach to hysteresis curves with one or more crossover points, several amendments would be possible. First, additional determination of the negative and positive maximum widths, both horizontally and vertically, as well as their positions provides information about the existence of a crossover point, as the difference will change its sign after a crossover point. If more than one crossover point is present, the algorithm must be executed on distinct ranges of the curve that are defined by the position of the crossover points to determine the width of each individual loop. Additionally, the position of the crossover points themselves could be considered as features for signal evaluation.

\section{Conclusions}

We have shown that hysteresis induced by cyclic changes of the gate bias can be used very effectively to increase the selectivity of $\mathrm{SiC}$ gas sensitive field effect transistors allowing identification of $\mathrm{NH}_{3}, \mathrm{CO}, \mathrm{NO}$ and $\mathrm{CH}_{4}$ even in changing background compositions, i.e., dry and humid nitrogen and air. Furthermore, quantification by taking the width of the hysteresis into account is possible as shown for $\mathrm{NH}_{3}$ and CO. Changes in the background gas mixture lead to strong changes in the shape of the signal; however, gases can still be identified.

The sweep rate of the applied gate bias ramp also has a strong influence on the signal shape that is strongly dependent on the gas and the sensor temperature. In any case, diffusion processes in the sensor and also chemical reactions on the sensor surface are accelerated at higher operating temperature, thus increasing the signal hysteresis and allowing one full measurement per minute. Easily computable features have been suggested and their application was demonstrated in a LDA to discriminate $500 \mathrm{ppm} \mathrm{CO}, \mathrm{NH}_{3}$ and dry nitrogen.

Future work will address the optimization of the GBCO technique both as stand-alone method as well as in combination with TCO to allow improved qualitative as well as quantitative measurements. This includes both the efficient determination of the optimum operating cycle for a given application as well as the optimum signal evaluation, i.e., obtaining the maximum information with the minimum of effort. Especially gas mixtures and dynamic gas composition changes need to be addressed if this approach is to be used in real applications, but also the signal stability needs to be taken into account.

Finally, the presented method of studying the sensor response can help to achieve a better understanding of gas interaction on and signal generation in GasFETs as the dynamic operation allows studying different effects on and in the sensor by choosing different temperatures and bias ramp rates to identify and separate different effects.

Acknowledgements. The authors would like to thank SenSiC $\mathrm{AB}$, Kista, Sweden, for providing the sensors and 3S - Sensors, Signal Processing, Systems GmbH, Saarbrücken, Germany, for providing the hardware for sensor operation and read-out.

Edited by: M. Penza

Reviewed by: two anonymous referees

\section{References}

Andersson, M., Pearce, R., and Lloyd Spetz, A.: New generation $\mathrm{SiC}$ based field effect transistor gas sensors, Sens. Actuators B., 179, 95-106, 2013.

Bastuck, M., Bur, C., Lloyd Spetz, A., Andersson, M., and Schütze, A.: Identification of ammonia and carbon monoxide based on the hysteresis of a gas-sensitive SiC field effect transistor, Proc. Transducers 2013, Barcelona, Spain, 2013.

Bur, C., Andersson, M., Lloyd Spetz, A., and Schütze, A.: Influence of a changing gate bias on the sensing properties of $\mathrm{SiC}$ field effect gas sensors, Proc. IMCS 2012, Nuremberg, Germany, 140143, 2012a.

Bur, C., Reimann, P., Andersson, M., Schütze, A., and Lloyd Spetz, A.: Increasing the selectivity of Pt-gate $\mathrm{SiC}$ field effect gas sensors by dynamic temperature modulation, IEEE Sensors Journal, 12, 1906-1913, 2012b. 
Bur, C., Bastuck, M., Andersson, M., Lloyd Spetz, A., and Schütze, A.: Combination of Temperature Cycled And Gate Bias Cycled Operation To Enhance the Selectivity of SiC-FET Gas Sensors, Proc. Transducers 2013, Barcelona, Spain, 2041-2044, 2013.

Darmastuti, Z., Pearce, R., Lloyd Spetz, A., and Andersson, M.: The influence of gate bias and structure on the CO sensing performance of $\mathrm{SiC}$ based field effect sensors, Proc. IEEE Sensors 2011, Limerick, Ireland, 133-136, 2011.

Fogelberg, J., Lundström, I., and Petersson, L.-G.: Ammonia Dissociation on Oxygen Covered Palladium studied with a Hydrogen Sensitive Pd-MOS device, Physica Scripta, 35, 702-705, 1987.

Gutierrez-Osuna, R.: Pattern Analysis for Machine Olfaction: A Review, IEEE Sensors Journal, 2, 189-202, 2002.

Johansson, S., Österlund, L., and Kasemo, B.: CO oxidation bistability diagrams for $\mathrm{Pt} / \mathrm{CeO}$ and $\mathrm{Pt} / \mathrm{SiO} 2$ model catalysts prepared by electron-beam lithography, Journal of Catalysis, 201, 275-285, 2001.

Klecka, W. R.: Discriminant Analysis, in Quantitative applications in the social sciences, SAGE University Paper, ISBN: $0803914911,1980$.

Lee, A. P. and Reedy, B. J.: Temperature modulation in semiconductor gas sensing, Sens. Actuators B, 60, 35-42, 1999.

Lloyd Spetz, A., Huatari, J., Bur, C., Bjorklund, R., Lappalainen, J., Jantunen, H., Schütze, A., and Andersson, M.: Chemical sensor systems for emission control from combustions, Sens. Actuators B, 187, 184-190, 2013.

Lundström, I, Sundgren, H., Winquist, F., Eriksson, M., KrantzRülcker, C., and Lloyd Spetz, A.: Twenty-?ve years of field effect gas sensor research in Linköping, Sens. Actuators B., 121, 247262, 2007.

Mattmann, M., Helbling, T., Durrer, L., Roman, C., Pohle, R., Fleischer, M., and Hierold, C.: Hysteresis reduction and measurement range enhancement of carbon nanotube based $\mathrm{NO}_{2}$ gas sensors by pulsed gate voltages, Procedia Chemistry, 1, 1431-1434, 2009.
Nakagomi, S., Tobias, P., Baranzahi, A., Lundström, I., Mårtensson, P., and Lloyd Spetz, A.: Influence of carbon monoxide, water and oxygen on high temperature catalytic metal-oxide-silicon carbide structures, Sens. Actuators B, 45, 183-191, 1997.

Nakagomi, S., Fukumura, A., Kokubun, Y., Savage, S., Wingbrant, H., Andersson, M., Lundström, I., Löfdahl, M., and Lloyd Spetz, A.: Influence of gate bias of MISiC-FET gas sensor device on the sensing properties, Sens. Actuators B, 108, 501-507, 2005.

Paska, Y. and Haick, H.: Interactive effect of hysteresis and surface chemistry on gated silicon nanowire gas sensors, ACS Appl. Mater. Interfaces, 4, 2604-2617, 2012.

Petit, C., Zander, D., Lmimouni, K., Ternisien, M., Tondelier, D., Lenfant, S., and Vuillaume, D.: Gate pulse electrical method to characterize hysteresis phenomena in organic field effect transistor, Org. Electron., 9, 979-984, 2008.

Schalwig, J., Kreisl, P., Ahlers, S., and Müller, G.: Response mechanism of SiC-based MOS field-effect gas sensors, IEEE Sensors Journal, 2, 394-402, 2002.

Schmidt-Zhang, P., Sandow, K.-P., Adolf, F., Göpel, W., and Guth, U.: A novel thick film sensor for simultaneous $\mathrm{O}_{2}$ and $\mathrm{NO}$ monitoring in exhaust gases, Sens. Actuators B, 70, 25-29, 2000.

Schütze, A., Gramm, A., and Rühl, T.: Identification of organic solvents by a virtual multisensor system with hierarchical classification, IEEE Sensors Journal, 4, 857-863, 2004.

Wallin, M., Grönbeck, H., Lloyd Spetz, A., and Skoglundh, M.: Vibrational study of ammonia adsorption on $\mathrm{Pt} / \mathrm{SiO}_{2}$, Appl. Surf. Sci., 235, 487-500, 2004. 